\title{
Synthesis and Antibacterial Activities of New 2-(Benzylthio)pyrimidines and 2-(Benzimidazolylmethylthio)pyrimidines Derivatives
}

\author{
Achi Patrick-Armand ${ }^{1}$, Coulibali Siomenan ${ }^{*}$, Zon Doumade ${ }^{2}$, Timotou Adéyolée ${ }^{2}$ Bolou Eric ${ }^{3}$, \\ Touré Daouda ${ }^{4}$, Sissouma Drissa' ${ }^{1}$, Adjou Ané ${ }^{1}$
}

${ }^{1}$ Laboratoire de Constitution et Réaction de la Matière, UFR Sciences des Structures de la Matière et Technologie, Université Félix Houphouët Boigny de Cocody, Abidjan, Côte d'Ivoire

${ }^{2}$ Département de Mathématiques-Physique-Chimie, UFR des Sciences Biologiques, Université Peleforo Gon Coulibaly, Korhogo, Côte d'Ivoire

${ }^{3}$ Centre National de Floristique, Université Félix Houphouët-Boigny de Cocody, Abidjan, Côte d'Ivoire

${ }^{4}$ Département de Biochimie-Génétique, UFR des Sciences Biologiques, Université Peleforo Gon Coulibaly, Korhogo, Côte d'Ivoire

Email: *bsiomenan@yahoo.fr

How to cite this paper: Patrick-Armand, A., Siomenan, C., Doumade, Z., Adéyolé, T., Eric, B., Daouda, T., Drissa, S. and Ané, A. (2021) Synthesis and Antibacterial Activities of New 2-(Benzylthio)pyrimidines and 2(Benzimidazolylmethylthio)pyrimidines Derivatives. Open Journal of Medicinal Chemistry, 11, 27-39.

https://doi.org/10.4236/ojmc.2021.113003

Received: June 29, 2021

Accepted: September 15, 2021

Published: September 18, 2021

Copyright $\odot 2021$ by author(s) and Scientific Research Publishing Inc.

This work is licensed under the Creative Commons Attribution International License (CC BY 4.0).

http://creativecommons.org/licenses/by/4.0/

(c) (i) Open Access

\begin{abstract}
A series of 2-(benzylthio)pyrimidines (6a-1) and 2-(benzimidazolylmethylthio)pyrimidines derivatives ( $6 \mathrm{~m}$ and $6 \mathrm{n}$ ) analogues of ethyl 2-(benzylthio)-6-methyl4-phenyl-1,4-dihydropyrimidine-5-carboxylates and ethyl 2-((1H-benzimidazol2-yl)methyl)thio)-6-methyl-4-phenyl-1,4-dihydropyri-midine-5-carboxylates were prepared and evaluated for antibacterial activity. These compounds were obtained by condensation of 2-thiopyrimidines (4) with benzyl halides or 2(chloromethyl)-1H-benzimidazole (5) in the presence of a base. All compounds were characterized by ${ }^{1} \mathrm{H},{ }^{13} \mathrm{C}$ and HRMS spectra. Out of fourteen, only eight compounds were screened against multi-resistant strains of Escherichia coli and Staphylococcus aureus. The results revealed that all of them were found to possess significant antibacterial activity against the germs tested. Compounds $6 \mathrm{c}, 6 \mathrm{~d}, 6 \mathrm{~h}$ and $6 \mathrm{~m}$ were more active on $S$. aureus and compounds $6 \mathrm{~h}$ and $6 \mathrm{~m}$ more active on $E$. coli.
\end{abstract}

\section{Keywords}

2-Thiopyrimidine, Benzylthiouracil, Benzimidazole, Antibacterial Activity

\section{Introduction}

The Pyrimidine ring is a well-known heterocyclic compound found in the struc- 
ture such as Uracil, cytosine and thymine. These bases are part of the composition of nucleosides forming DNA and RNA, like Uridine, which has Uralic as a nitrogen base (Figure 1). This Pyrimidine ring is part of a very important new class of heterocyclic compounds widely used as building blocks of pharmaceutical agents [1] [2]. It has many biological properties namely antiviral [3], antimicrobial [4], anti-tuberculosis [5], analgesic [6] and also is used as an antihypertensive [7] and antitumor agent [8]. Studies showed that poly-substituted Pyrimidines could be used as potential anti-tumor agents [9] [10] [11]. Indeed, the sulfur derivatives of Uracil which gave 2-thiouracils are used as anti-inflammatory and virucidal agents [12]. Figure 1 shows 2 -thiopyrimidines derivatives resulting from the replacement of the oxygen atom bonded to C-2 in the uridine base [13] by a sulfur atom and the replacement of the oxygen in the carbon C-4 by a phenyl group. A European patent [14] has revealed the importance of 2-thiopyrimdines derivatives in the preparation of cardiotonic drugs. Benzylthiouracil marketed under the name BASDENE is a synthetic antithyroid drug. Pathak et al. have evaluated and reported the existence of a primary activity of 2-thiopyrimidine derivatives against Mycobacterium tuberculosis [15]. Moreover, Biginelli in 1893, synthesized 2-thiopyrimidines [16] which demonstrated several pharmaceutical properties. The 2-thiopyrimidines was found to have excellent antimicrobial [17] [18] [19], antiviral [20], anti-inflammatory [21] and antitumor [22] properties. Therefore, 2-thiopyrimidines gained more attention from worldwide organic chemists [23]. Furthermore, the benzimidazole ring also had a panel of biological activities [24] [25] [26]. Among them, the most important which could be quoted are the antiviral [24], antiplasmodial [25] and anthelmintic [26] (Figure 1). Thus, pyrimidines or thiopyrimidines and benzimidazoles play an important role in cellular processes making these molecules valuable to become leads for new drug discoveries. To investigate new antimicrobial agents, we initiated the synthesis of new pyrimidines derivatives attaching benzylthio and benzimidazolylthio groups in position- 2 and then evaluated their antibacterial activity.

\section{Materials and Methods}

\subsection{Materials}

\subsubsection{Materials of Chemistry}

Unless otherwise indicated, ${ }^{1} \mathrm{H}$ and ${ }^{13} \mathrm{C}$ NMR spectra were recorded at 300 and $75 \mathrm{MHz}$ or 400 and $101 \mathrm{MHz}$ or 600 and $151 \mathrm{MHz}$, respectively, in $\mathrm{CDCl}_{3}$, DMSO- $d_{6}$ and Acetone- $d_{6}$ solutions. For ${ }^{1} \mathrm{H}$ NMR assignments, the chemical shifts are reported in ppm on the $\boldsymbol{\delta}$ scale. The following notation is used for the ${ }^{1} \mathrm{H}$ NMR spectral splitting patterns: s (singlet), d (doublet), dd (doublet of doublet), $\mathrm{t}$ (triplet), $\mathrm{q}$ (quadruplet), $\mathrm{m}$ (multiplet) and further qualified as app (apparent), br (broad) coupling constants, Jare reported in Hz. HRMS were measured in the electrospray (ESI) mode on a LC-MSD TOF mass analyzer.

\subsubsection{Biological Materials \\ Microbial strains}


<smiles></smiles><smiles>O=c1cc(Cc2ccccc2)[nH]c(=S)[nH]1</smiles>

Benzylthiouracile Basdène $\mathbb{R}$<smiles>[R]c1ccc(C2NC(=S)NC(C)=C2C(=O)OCC)cc1</smiles>

$\mathrm{R}=\mathrm{H}, \mathrm{CH}_{3}$ 2-thiopyrimidines<smiles>[R]c1nc2ccccc2n1P</smiles>

Benzimidazole

Figure 1. Structures of pyrimidine and benzimidazole biological molecules.

The microbial support was composed of clinical strains of E. Coli (Gram negative bacteria) and $S$. aureus (Gram positive bacteria). Strains were provided by the National Public Health Laboratory (LNSP) of Ivory Coast. These strains are all pathogenic and multi-resistants. Strains of $S$. aureus are resistant to: Amoxicillin, Ampicillin, Oxacillin, Ceftazidine, Fosfomycin, Vancomycin and Cefsulodine and those of $E$. coli were all resistant to: Amoxicillin, Ampicillin, Ceftriazone, Fosfomycin, Cefsulodin. The culture medium used was Mueller-Hinton broth (Oxoid) and Mueller-Hinton agar (Lab.Conda s.a). The synthetic compounds used in the experiments were eight 2-thiopyrimidine derivatives $(6 \mathrm{a}, 6 \mathrm{~b}, 6 \mathrm{c}, 6 \mathrm{~d}, 6 \mathrm{~h}, 6 \mathrm{j}, 6 \mathrm{k}$, $6 \mathrm{~m})$. Dimethylsulfoxide (DMSO) and distilled water were used as solvents for the solubilization of chemicals.

\subsection{Methods}

\subsubsection{Methods of Synthesis}

\section{Method of synthesis of compounds $4 \mathrm{a}$ and $4 \mathrm{~b}$}

$12.5 \mathrm{mmol}$ of thiourea, $13 \mathrm{mmol}$ of benzaldehyde and $19 \mathrm{mmol}$ of ethyl acetoacetate were dissolved in $10 \mathrm{~mL}$ of anhydrous ethanol and then ten drops of concentrated hydrochloric acid (37\%) were added. The reaction mixture was stirred under reflux in ethanol for $2 \mathrm{~h}$. After that time the reaction was quenched by addition of ice water. The resulting white precipitate obtained which was filtered and then washed with cold ethanol. The crystals obtained were purified by recrystallization in ethanol.

\section{General procedure for the synthesis of compounds 6a-1}

$1 \mathrm{mmol}$ of the 2-thiopyrimidine derivative was dissolved in $10 \mathrm{~mL}$ of dimethylformamide (DMF), then $1.5 \mathrm{mmol}$ of potassium carbonate $\left(\mathrm{K}_{2} \mathrm{CO}_{3}\right)$ were added. The reaction was stirred at room temperature and then $1.3 \mathrm{mmol}$ of substituted benzyl chloride or bromide were added dropwise.

From $2 \mathrm{~h}$ to overnight, the reaction mixture was neutralized with a dilute solution of $\mathrm{HCl}(2 \mathrm{M})$. The precipitate formed was filtered and purified by silica gel chromatography with a mixture of ethyl acetate/ hexane. Compounds 6a-1 was obtained with yields between $50 \%$ to $94 \%$ yields.

Method of synthesis of compounds $6 \mathrm{~m}$ and $6 \mathrm{n}$

$1.81 \mathrm{mmol}$ of 2-thiopyrimidine were dissolved in $25 \mathrm{~mL}$ of tetrahydrofuran 
(THF), then $2.89 \mathrm{mmol}$ of triethylamine $\left(\mathrm{Et}_{3} \mathrm{~N}\right)$ were added. The mixture was stirred at room temperature for $15 \mathrm{~min}$, then $2.2 \mathrm{mmol}$ of 2-(chloromethyl)$1 H$-benzimidazole were added dropwise. After $24 \mathrm{~h}$, solvent was evaporated and the organic layer was concentrated under vacuo. Then ice was added to the mixture. The precipitate formed was filtered and purified by silica gel chromatography with a mixture of ethyl acetate/ hexane. Compounds $6 \mathrm{~m}$ and $6 \mathrm{n}$ were obtained with $60 \%$ and $55 \%$ yields respectively.

Products characterizations

\section{Ethyl}

2-(benzylthio)-6-methyl-4-phenyl-1,4-dihydropyrimidine-5-carboxylate 6a

Yield = 62\%; ${ }^{1} \mathrm{H}$ NMR (300 MHz, $\left.\mathrm{CDCl}_{3}\right) \delta(\mathrm{ppm}) 11.20(\mathrm{~s}, 1 \mathrm{H}, \mathrm{NH}), 7.36-$ $6.93\left(\mathrm{~m}, 10 \mathrm{H}, \mathrm{H}_{\mathrm{Ar}}\right), 5.82(\mathrm{~s}, 1 \mathrm{H}, \mathrm{CH}), 4.91\left(\mathrm{~d}, \mathrm{~J}_{a b}=13.4 \mathrm{~Hz}, 1 \mathrm{H}_{\mathrm{a}}, \mathrm{S}-\mathrm{CH}_{2}\right), 4.28(\mathrm{~d}$, $\left.J_{b a}=13.4 \mathrm{~Hz}, 1 \mathrm{H}_{\mathrm{b}}, \mathrm{S}-\mathrm{CH}_{2}\right), 4.22-3.82\left(\mathrm{~m}, 2 \mathrm{H}, \mathrm{O}-\mathrm{CH}_{2}\right), 2.57\left(\mathrm{~s}, 3 \mathrm{H}, \mathrm{CH}_{3}\right), 1.14(\mathrm{t}$, $\left.J=7.1 \mathrm{~Hz}, 3 \mathrm{H}, \mathrm{CH}_{3}\right) ;{ }^{13} \mathrm{C} \mathrm{NMR}\left(75 \mathrm{MHz}, \mathrm{CDCl}_{3}\right) \delta(\mathrm{ppm}): 164.01,161.00$, $142.95,139.64,132.94,129.10,129.03,128.98,128.51,127.28,106.20,61.05$, 54.71, 37.34, 17.28, 13.90. HRMS (ESI): Calc for $\mathrm{C}_{21} \mathrm{H}_{23} \mathrm{~N}_{2} \mathrm{O}_{2} \mathrm{~S}(\mathrm{M}+\mathrm{H})^{+}: 367.1576$. Found: 367.1570 .

Ethyl 2-((4-chlorobenzyl)

thio)-6-methyl-4-phenyl-1,4-dihydropyrimidine-5-carboxylate $6 b$

Yield = 94\%; ${ }^{1} \mathrm{H}$ NMR (300 MHz, DMSO- $\left.d_{6}\right) \delta(\mathrm{ppm}) 7.56-6.89(\mathrm{~m}, 9 \mathrm{H}$, $\mathrm{H}_{\mathrm{Ar}}$ ), $5.59(\mathrm{~s}, 1 \mathrm{H}, \mathrm{CH}), 4.90\left(\mathrm{~d}, J_{a b}=16.3 \mathrm{~Hz}, 1 \mathrm{H}_{\mathrm{a}}, \mathrm{S}-\mathrm{CH}_{2}\right), 4.38\left(\mathrm{~d}, J_{b a}=16.3 \mathrm{~Hz}\right.$, $\left.1 \mathrm{H}_{\mathrm{b}}, \mathrm{S}-\mathrm{CH}_{2}\right), 4.10-3.99\left(\mathrm{~m}, 2 \mathrm{H}, \mathrm{CH}_{2}-\mathrm{O}\right), 2.38\left(\mathrm{~s}, 3 \mathrm{H}, \mathrm{CH}_{3}\right), 1.08$ (t, J=7.1 Hz, $3 \mathrm{H}, \mathrm{CH}_{3}$ ); ${ }^{13} \mathrm{C}$ NMR (75 MHz, DMSO- $\left.d_{6}\right) \delta$ (ppm) 164.55, 144.31, 140.62, $135.11,132.87,130.96,129.33$, 128.92, 127.24, 104.89, 60.97, 54.98, 34.66, 17.42, 14.30; HRMS (ESI): Calc for $\mathrm{C}_{21} \mathrm{H}_{22} \mathrm{ClN}_{2} \mathrm{O}_{2} \mathrm{~S}(\mathrm{M}+\mathrm{H})^{+}$: 401.1017. Found: 401.1015.

Ethyl 6-methyl-2-((3-nitrobenzyl)

thio)-4-phenyl-1,4-dihydropyrimidine-5-carboxylate $6 \mathrm{c}$

Yield $=50 \%,{ }^{1} \mathrm{H}$ NMR (300 $\left.\mathrm{MHz} \mathrm{CDCl}_{3}\right) \delta(\mathrm{ppm}): 8.17\left(\mathrm{~s}, 1 \mathrm{H}, \mathrm{H}_{\mathrm{Ar}}\right), 8.04(\mathrm{~d}$, $\left.J=8.0 \mathrm{~Hz}, 1 \mathrm{H}, \mathrm{H}_{\mathrm{Ar}}\right), 7.57\left(\mathrm{~s}, 1 \mathrm{H}, \mathrm{H}_{\mathrm{Ar}}\right), 7.34\left(\mathrm{~s}, 2 \mathrm{H}, \mathrm{H}_{\mathrm{Ar}}\right), 7.31-7.11\left(\mathrm{~m}, 4 \mathrm{H}, \mathrm{H}_{\mathrm{Ar}}\right)$, $5.56(\mathrm{~s}, 1 \mathrm{H}, \mathrm{CH}), 5.43\left(\mathrm{~d}, J=4.5 \mathrm{~Hz}, 1 \mathrm{H}_{\mathrm{a}}, \mathrm{S}-\mathrm{CH}_{2}\right), 4.21-4.01\left(\mathrm{~m}, 3 \mathrm{H}, \mathrm{H}_{\mathrm{b}}\right.$, $\mathrm{CH}_{2}-\mathrm{O}$ ), $2.38\left(\mathrm{~s}, 3 \mathrm{H}, \mathrm{CH}_{3}\right), 1.19\left(\mathrm{t}, J=7.1 \mathrm{~Hz}, 3 \mathrm{H}, \mathrm{CH}_{3}\right) . ;{ }^{13} \mathrm{C} \mathrm{NMR}$ (75 MHz, $\left.\mathrm{CDCl}_{3}\right) \delta(\mathrm{ppm}) 166.59,165.65,153.28,148.12,146.27,143.70,140.16,135.24$, $129.21,128.73,128.42,127.98,126.94,126.62,123.99,122.24,101.17,60.06$, 55.40, 33.64, 18.59, 14.29.; HRMS (ESI): Calc for $\mathrm{C}_{21} \mathrm{H}_{22} \mathrm{~N}_{3} \mathrm{O}_{4} \mathrm{~S}(\mathrm{M}+\mathrm{H})^{+}: 412.1716$. Found: 412.1716.

Ethyl 6-methyl-2-((4-methylbenzyl) thio)-4-phenyl-1,4-dihydropyrimidine-5-carboxylate $6 d$

Yield $=57 \%,{ }^{1} \mathrm{H}$ NMR (400 MHz, $\left.\mathrm{CDCl}_{3}\right) \delta(\mathrm{ppm}) 7.36-7.27\left(\mathrm{~m}, 4 \mathrm{H}, \mathrm{H}_{\mathrm{Ar}}\right)$, $7.19-7.11\left(\mathrm{~m}, 5 \mathrm{H}, \mathrm{H}_{\mathrm{Ar}}\right), 5.25(\mathrm{~s}, 1 \mathrm{H}, \mathrm{CH}), 4.90\left(\mathrm{~d}, J_{a b}=13.3 \mathrm{~Hz}, 1 \mathrm{H}_{\mathrm{a}}, \mathrm{S}-\mathrm{CH}_{2}\right)$, $4.41\left(\mathrm{~d}, \mathrm{~J}_{b a}=13.3 \mathrm{~Hz}, 1 \mathrm{H}_{\mathrm{b}}, \mathrm{S}-\mathrm{CH}_{2}\right), 4.14-3.97\left(\mathrm{~m}, 2 \mathrm{H}, \mathrm{O}-\mathrm{CH}_{2}\right), 2.39(\mathrm{~s}, 3 \mathrm{H}$, $\left.\mathrm{CH}_{3}\right), 1.16\left(\mathrm{t}, J=9.2 \mathrm{~Hz}, 3 \mathrm{H}, \mathrm{CH}_{3}\right) ;{ }^{13} \mathrm{C} \mathrm{NMR}\left(101 \mathrm{MHz}, \mathrm{CDCl}_{3}\right) \delta(\mathrm{ppm})$ 166.72 , 161.63, 154.80, 142.40, 137.64, 137.16, 134.38, 132.37, 129.47, 129.21, $129.14,128.54,128.01,127.49,59.73,51.78,36.28,23.44,21.19,14.20$; HRMS 
(ESI): Calc for $\mathrm{C}_{22} \mathrm{H}_{25} \mathrm{~N}_{2} \mathrm{O}_{2} \mathrm{~S}(\mathrm{M}+\mathrm{H})^{+}:$381.1508. Found: 381.1506 .

Ethyl 2-((3,5-dichlorobenzyl)

thio)-6-methyl-4-phenyl-1,4-dihydropyrimidine-5-carboxylate $6 e$

Yield $=51 \%,{ }^{1} \mathrm{H}$ NMR $\left(600 \mathrm{MHz}, \mathrm{CDCl}_{3}\right) \delta(\mathrm{ppm}) 7.28-7.11\left(\mathrm{~m}, 9 \mathrm{H}, \mathrm{H}_{\mathrm{A}} \mathrm{r}\right)$, $5.74(\mathrm{~s}, 1 \mathrm{H},-\mathrm{CH}), 4.77\left(\mathrm{~d}, J_{a b}=12.3 \mathrm{~Hz}, 1 \mathrm{H}_{a}, \mathrm{~S}-\mathrm{CH}_{2}\right), 4.16\left(\mathrm{~d}, J_{b a}=12.3 \mathrm{~Hz}, 1 \mathrm{H}_{\mathrm{b}}\right.$, S- $\left.\mathrm{CH}_{2}\right), 4.13-4.06\left(\mathrm{~m}, 2 \mathrm{H}, \mathrm{CH}_{2}-\mathrm{O}\right), 2.51\left(\mathrm{~s}, 3 \mathrm{H}, \mathrm{CH}_{3}\right), 1.17(\mathrm{t}, J=7.1 \mathrm{~Hz}, 3 \mathrm{H}$, $\left.\mathrm{CH}_{3}\right) ;{ }^{13} \mathrm{C}$ NMR (151 MHz, $\left.\mathrm{CDCl}_{3}\right) ; \delta$ (ppm) 165.12, 145.15, 141.30, 139.09, 135.01, 128.79, 128.42, 128.23, 127.37, 126.74, 104.72, 60.63, 34.52, 14.10.; HRMS (ESI): Calc for $\mathrm{C}_{21} \mathrm{H}_{20} \mathrm{Cl}_{2} \mathrm{~N}_{2} \mathrm{NaO}_{2} \mathrm{~S}(\mathrm{M}+\mathrm{Na})^{+}$: 457.0725. Found: 457.0729.

Ethyl 2-((4-(methoxycarbonyl) benzyl) thio)-6-methyl-4-phenyl-1,4-dihydropyrimidine-5-carboxylate $6 f$

Yield $=59 \%,{ }^{1} \mathrm{H}$ NMR $\left(400 \mathrm{MHz}, \mathrm{CDCl}_{3}\right) \delta(\mathrm{ppm}) 7.95-7.75\left(\mathrm{~m}, 4 \mathrm{H}, \mathrm{H}_{\mathrm{Ar}}\right)$, $7.39-7.17\left(\mathrm{~m}, 5 \mathrm{H}, \mathrm{H}_{\mathrm{Ar}}\right), 5.66(\mathrm{~s}, 1 \mathrm{H},-\mathrm{CH}-), 4.47\left(\mathrm{~d}, J_{a b}=6.9 \mathrm{~Hz}, 1 \mathrm{H}_{\mathrm{a}}, \mathrm{S}_{-} \mathrm{CH}_{2}\right)$, $4.14\left(\mathrm{~d}, J_{b a}=6.9 \mathrm{~Hz}, 1 \mathrm{H}_{\mathrm{b}}, \mathrm{S}-\mathrm{CH}_{2}\right), 4.13-4.06\left(\mathrm{~m}, 2 \mathrm{H}, \mathrm{CH}_{2}-\mathrm{O}\right), 3.91(\mathrm{~s}, 3 \mathrm{H}$, $\mathrm{CH}_{3}-\mathrm{O}$ ), $2.32\left(\mathrm{~s}, 3 \mathrm{H}, \mathrm{CH}_{3}\right), 1.2\left(\mathrm{t}, J=7.1 \mathrm{~Hz}, 3 \mathrm{H}, \mathrm{CH}_{3}\right) ;{ }^{13} \mathrm{C}$ NMR $(101 \mathrm{MHz}$, $\left.\mathrm{CDCl}_{3}\right) \delta(\mathrm{ppm}) 166.93,166.73,144.49,143.15,128.71,128.39,128.00,126.96$, 101.65, 59.86, 52.36, 34.64, 22.8, 14.37; HRMS(ESI): Calc for $\mathrm{C}_{23} \mathrm{H}_{24} \mathrm{~N}_{2} \mathrm{NaO}_{4} \mathrm{~S}$ $(\mathrm{M}+\mathrm{Na})^{+}:$447.1283. Found: 447.1285.

\section{Ethyl}

2-(benzylthio)-6-methyl-4-(p-tolyl)-1,4-dihydropyrimidine-5-carboxylate $6 g$

Yield $=51 \%,{ }^{1} \mathrm{H}$ NMR $\left(300 \mathrm{MHz}, \mathrm{CDCl}_{3}\right) \delta(\mathrm{ppm}) 7.50-6.67\left(\mathrm{~m}, 9 \mathrm{H}, \mathrm{H}_{\mathrm{Ar}}\right)$, $5.68(\mathrm{~s}, 1 \mathrm{H}, \mathrm{CH}), 4.36\left(\mathrm{~d}, J_{a b}=13.4 \mathrm{~Hz}, 1 \mathrm{H}_{\mathrm{a}}, \mathrm{S}-\mathrm{CH}_{2}\right), 4.13-4.09\left(\mathrm{~m}, 3 \mathrm{H}, \mathrm{H}_{\mathrm{b}}\right.$, $\mathrm{CH}_{2}-\mathrm{O}$ ), 2.33 (s, 6H, $2 \mathrm{CH}_{3}$ ), $1.23\left(\mathrm{t}, J=7.1 \mathrm{~Hz}, 3 \mathrm{H}, \mathrm{CH}_{3}\right) ;{ }^{13} \mathrm{C}$ NMR $(75 \mathrm{MHz}$, $\left.\mathrm{CDCl}_{3}\right) \delta(\mathrm{ppm}) 167.02,160.03,146.63,134.56,129.36,129.24,128.93,119.51$, 112.52, 60.07, 54.71, 35.04, 21.29, 14.29.; HRMS(ESI): Calc for $\mathrm{C}_{22} \mathrm{H}_{25} \mathrm{~N}_{2} \mathrm{O}_{2} \mathrm{~S}$ $(\mathrm{M}+\mathrm{H})^{+}:$381.1839. Found: 381.1842 .

Ethyl 2-((4-chlorobenzyl) thio)-6-methyl-4-(p-tolyl)-1,4-dihydropyrimidine-5-carboxylate $6 \mathrm{~h}$

Yield $=75 \%,{ }^{1} \mathrm{H}$ NMR $\left(300 \mathrm{MHz}, \mathrm{CDCl}_{3}\right) \delta(\mathrm{ppm}) 7.26-7.04\left(\mathrm{~m}, 8 \mathrm{H}, \mathrm{H}_{\mathrm{Ar}}\right)$, $5.63(\mathrm{~s}, 1 \mathrm{H},-\mathrm{CH}), 4.36\left(\mathrm{~d}, J_{a b}=13.6 \mathrm{~Hz}, 1 \mathrm{H}_{\mathrm{a}}, \mathrm{S}-\mathrm{CH}_{2}\right), 4.13\left(\mathrm{~d}, J_{b a}=13.6 \mathrm{~Hz}, 1 \mathrm{H}\right.$, S- $\left.\mathrm{CH}_{2}\right), 4.10-4.00\left(\mathrm{~m}, 2 \mathrm{H}, \mathrm{CH}_{2}-0\right), 2.36\left(\mathrm{~s}, 6 \mathrm{H}, 2 \mathrm{CH}_{3}\right), 1.22(\mathrm{t}, J=7.1 \mathrm{~Hz}, 3 \mathrm{H}$, $\left.\mathrm{CH}_{3}\right) ;{ }^{13} \mathrm{C}$ NMR (75 $\mathrm{MHz}, \mathrm{CDCl}_{3}$ ) $\delta(\mathrm{ppm}) 167.04,141.98,136.26,133.22$, 130.37, 129.10, 128.54, 126.91, 60.06, 34.38, 21.28, 14.26.; HRMS(ESI): Calc for $\mathrm{C}_{22} \mathrm{H}_{23} \mathrm{ClN}_{2} \mathrm{NaO}_{2} \mathrm{~S}(\mathrm{M}+\mathrm{Na})^{+}$: 437.0923. Found: 437.0920 .

Ethyl 6-methyl-2-((3-nitrobenzyl)

thio)-4-(p-tolyl)-1,4-dihydropyrimidine-5-carboxylate $6 i$

Yield $=65 \%,{ }^{1} \mathrm{H}$ NMR $\left(400 \mathrm{MHz}, \mathrm{CDCl}_{3}\right) \delta(\mathrm{ppm}) 8.16\left(\mathrm{~m}, 1 \mathrm{H}, \mathrm{H}_{\mathrm{Ar}}\right), 8.04$ $\left(\mathrm{d}, J=7.7 \mathrm{~Hz}, 1 \mathrm{H}, \mathrm{H}_{\mathrm{Ar}}\right), 7.58\left(\mathrm{dd}, J=8.2,6.8 \mathrm{~Hz}, 1 \mathrm{H}, \mathrm{H}_{\mathrm{Ar}}\right), 7.34-7.24(\mathrm{~m}, 1 \mathrm{H}$, $\left.\mathrm{H}_{\mathrm{Ar}}\right), 7.14\left(\mathrm{~d}, J=8.0 \mathrm{~Hz}, 2 \mathrm{H}, \mathrm{H}_{\mathrm{Ar}}\right), 7.05\left(\mathrm{~d}, J=7.8 \mathrm{~Hz}, 2 \mathrm{H}, \mathrm{H}_{\mathrm{Ar}}\right), 5.54(\mathrm{~S}, 1 \mathrm{H}$, $\mathrm{CH}), 4.49\left(\mathrm{~d}, \mathrm{~J}_{a b}=13.2 \mathrm{~Hz}, 1 \mathrm{H}_{\mathrm{a}}, \mathrm{S}-\mathrm{CH}_{2}\right), 4.22-4.01\left(\mathrm{~m}, 3 \mathrm{H}, \mathrm{H}_{\mathrm{b}}, \mathrm{CH}_{2}-\mathrm{O}\right)$ ), 2.32 (s, $\left.6 \mathrm{H}, 2 \mathrm{CH}_{3}\right), 1.20\left(\mathrm{t}, J=7.1 \mathrm{~Hz}, 3 \mathrm{H}, \mathrm{CH}_{3}\right) .{ }^{13} \mathrm{C} \mathrm{NMR}\left(101 \mathrm{MHz}, \mathrm{CDCl}_{3}\right) \delta$ (ppm) 166.72, 148.04, 141.55, 140.26, 135.22, 129.15, 126.81, 124.00, 122.13, 59.88, 33.97, 21.10, 14.21. HRMS (ESI): Calc for $\mathrm{C}_{22} \mathrm{H}_{24} \mathrm{~N}_{3} \mathrm{O}_{4} \mathrm{~S}(\mathrm{M}+\mathrm{H})^{+}$: 426.1938 . Found: 426.1942. 
Ethyl 6-methyl-2-((4-methylbenzyl)

thio)-4-(p-tolyl)-1,4-dihydropyrimidine-5-carboxylate $6 j$

Yield $=67 \%,{ }^{1} \mathrm{H}$ NMR (600 MHz, $\left.\mathrm{CDCl}_{3}\right) \delta(\mathrm{ppm}) 11.06(\mathrm{~s}, 1 \mathrm{H}, \mathrm{NH}), 7.14$ $\left(\mathrm{d}, J=7.9 \mathrm{~Hz}, 2 \mathrm{H}, \mathrm{H}_{\mathrm{Ar}}\right), 7.07\left(\mathrm{~d}, J=7.9 \mathrm{~Hz}, 2 \mathrm{H}, \mathrm{H}_{\mathrm{Ar}}\right), 7.01(\mathrm{~d}, J=7.9 \mathrm{~Hz}, 2 \mathrm{H}$, $\left.\mathrm{H}_{\mathrm{Ar}}\right), 6.84\left(\mathrm{~d}, J=7.9 \mathrm{~Hz}, 2 \mathrm{H}, \mathrm{H}_{\mathrm{Ar}}\right), 5.79(\mathrm{~s}, 1 \mathrm{H},-\mathrm{CH}-), 4.80\left(\mathrm{~d}, J_{a b}=13.3 \mathrm{~Hz}, 1 \mathrm{H}_{\mathrm{a}}\right.$, $\left.\mathrm{S}-\mathrm{CH}_{2}\right), 4.28\left(\mathrm{~d}, \mathrm{~J}_{b a}=13.3 \mathrm{~Hz}, 1 \mathrm{H}_{\mathrm{b}}, \mathrm{S}-\mathrm{CH}_{2}\right), 4.15-3.98\left(\mathrm{~m}, 2 \mathrm{H}, \mathrm{CH}_{2}-\mathrm{O}\right), 2.56$ (s, $\left.3 \mathrm{H}, \mathrm{CH}_{3}\right), 2.31\left(\mathrm{~s}, 3 \mathrm{H}, \mathrm{CH}_{3}\right), 2.23\left(\mathrm{~s}, 3 \mathrm{H}, \mathrm{CH}_{3}\right), 1.13\left(\mathrm{t}, J=7.1 \mathrm{~Hz}, 3 \mathrm{H}, \mathrm{CH}_{3}\right) . ;{ }^{13} \mathrm{C}$ NMR (151 MHz, $\left.\mathrm{CDCl}_{3}\right) \delta(\mathrm{ppm}) 163.95,161.35,143.03,138.68,138.11,136.74$, $129.75,129.57,129.44,128.97,127.20,106.07,60.94,54.41,36.88,21.23,21.16,17.29$, 14.01.; HRMS (ESI): Calc for $\mathrm{C}_{23} \mathrm{H}_{27} \mathrm{~N}_{2} \mathrm{O}_{2} \mathrm{~S}(\mathrm{M}+\mathrm{H})^{+}:$395.1276. Found: 395.1279.

Ethyl 2-((4-(3, 5-dichloro benzyl) thio)-6-methyl-4-(p-tolyl)-1,4-dihydropyrimidine-5-carboxylate $6 \mathrm{k}$

Yield $=59 \%,{ }^{1} \mathrm{H}$ NMR $\left(400 \mathrm{MHz}, \mathrm{DMSO}-d_{\sigma}\right) \delta(\mathrm{ppm}) 7.41(\mathrm{dd}, J=5.2,3.5$ $\left.H z, 1 \mathrm{H}, \mathrm{H}_{\mathrm{Ar}}\right), 7.33\left(\mathrm{~d}, \mathrm{~J}=1.6 \mathrm{~Hz}, 2 \mathrm{H}, \mathrm{H}_{\mathrm{Ar}}\right), 7.07\left(\mathrm{~d}, J=8.1 \mathrm{~Hz}, 2 \mathrm{H}, \mathrm{H}_{\mathrm{Ar}}\right), 6.99(\mathrm{~d}, J$ $\left.=8.1 \mathrm{~Hz}, 2 \mathrm{H}, \mathrm{H}_{\mathrm{Ar}}\right), 5.50(\mathrm{~s}, 1 \mathrm{H},-\mathrm{CH}), 4.99\left(\mathrm{~d}, J_{a b}=14.7 \mathrm{~Hz}, 1 \mathrm{H}_{\mathrm{a}}, \mathrm{S}-\mathrm{CH}_{2}\right), 4.37(\mathrm{~d}$, $\left.J_{b a}=14.7 \mathrm{~Hz}, 1 \mathrm{H}_{\mathrm{b}}, \mathrm{S}-\mathrm{CH}_{2}\right), 4.05\left(\mathrm{q}, J=7.1 \mathrm{~Hz}, 2 \mathrm{H}, \mathrm{CH}_{3}\right), 2.28\left(\mathrm{~s}, 3 \mathrm{H}, \mathrm{CH}_{3}\right), 2.09$ (s, 3H, $\left.\mathrm{CH}_{3}\right), 1.11$ (t, $\left.J=7.1 \mathrm{~Hz}, 3 \mathrm{H}, \mathrm{CH}_{3}\right) ;{ }^{13} \mathrm{C}$ NMR (101 MHz, DMSO-d6) $\delta$ (ppm) 165.85, 163.25, 136.46, 134.76, 132.57, 131.44, 130.97, 129.20, 127.90, 126.69, 106.07, 65.94, 59.41, 31.07, 22.26, 18.11, 14.41; HRMS(ESI): Calc for $\mathrm{C}_{22} \mathrm{H}_{23} \mathrm{Cl}_{2} \mathrm{~N}_{2} \mathrm{O}_{4} \mathrm{~S}(\mathrm{M}+\mathrm{H})^{+}:$449.2329. Found: 449.2327.

Ethyl 2-((4-(methoxycarbonyl) benzyl)

thio)-6-methyl-4-(p-tolyl)-1,4-dihydropyrimidine-5-carboxylate 61

Yield $=55 \%,{ }^{1} \mathrm{H}$ NMR $\left(300 \mathrm{MHz}, \mathrm{CDCl}_{3}\right) \delta(\mathrm{ppm}) 11.26(\mathrm{~s}, 1 \mathrm{H}, \mathrm{NH}), 7.66$ $\left(\mathrm{d}, J=8.0 \mathrm{~Hz}, 2 \mathrm{H}, \mathrm{H}_{\mathrm{Ar}}\right), 7.40-7.20\left(\mathrm{~m}, 2 \mathrm{H}, \mathrm{H}_{\mathrm{Ar}}\right), 7.07\left(\mathrm{~d}, J=8.0 \mathrm{~Hz}, 2 \mathrm{H}, \mathrm{H}_{\mathrm{Ar}}\right)$, $6.98\left(\mathrm{~d}, J=7.9 \mathrm{~Hz}, 2 \mathrm{H}, \mathrm{H}_{\mathrm{Ar}}\right.$ ), 5.77 (s, $\left.1 \mathrm{H},-\mathrm{CH}-\right), 5.22$ (d, $J_{a b}=14.3 \mathrm{~Hz}, 1 \mathrm{H}_{\mathrm{a}}$, $\left.\mathrm{S}-\mathrm{CH}_{2}\right), 4.21\left(\mathrm{~d}, \mathrm{~J}_{b a}=14.3 \mathrm{~Hz}, 1 \mathrm{H}_{\mathrm{b}}, \mathrm{S}-\mathrm{CH}_{2}\right), 4.13-3.98\left(\mathrm{~m}, 2 \mathrm{H}, \mathrm{CH}_{2}-\mathrm{O}\right), 3.92(\mathrm{~s}$, $3 \mathrm{H}, \mathrm{CH}_{3}-\mathrm{O}$ ), 2.59 (s, 3H, CH${ }_{3}$ ), 2.29 (s, 3H, $\left.\mathrm{CH}_{3}\right), 1.13$ (t, J = $7.1 \mathrm{~Hz}, 3 \mathrm{H}, \mathrm{CH}_{3}$ ); ${ }^{13} \mathrm{C} \mathrm{NMR}\left(75 \mathrm{MHz}, \mathrm{CDCl}_{3}\right) \delta(\mathrm{ppm}) 166.23,163.82,160.47,142.69,139.13$, $138.51,136.31,129.93,129.82,129.70,128.93,127.14,106.46,61.13,54.65,52.19$, 36.40, 21.09, 17.31, 14.02; HRMS (ESI): Calc for $\mathrm{C}_{24} \mathrm{H}_{27} \mathrm{~N}_{2} \mathrm{O}_{4} \mathrm{~S}(\mathrm{M}+\mathrm{H})^{+}$: 439.1232 . Found: 439.1229 .

Ethyl 2-(((1H-benzo[d]imidazol-2-yl)

methyl)thio)-6-methyl-4-phenyl-1,4-dihydropyrimidine-5-carboxylate $6 \mathrm{~m}$

Yield $=60 \%,{ }^{1} \mathrm{H}$ NMR $\left(300 \mathrm{MHz}, \mathrm{CDCl}_{3}\right): \delta(\mathrm{ppm}) 7.51-7.15\left(\mathrm{~m}, 9 \mathrm{H}, \mathrm{H}_{\mathrm{Ar}}\right)$, $5.80(\mathrm{~s}, 1 \mathrm{H}, \mathrm{CH}), 4.53\left(\mathrm{~d}, J_{a b}=14.7 \mathrm{~Hz}, 1 \mathrm{H}_{\mathrm{a}}, \mathrm{S}-\mathrm{CH}_{2}\right), 4.22-4.07\left(\mathrm{~m}, 2 \mathrm{H}, 1 \mathrm{H}_{\mathrm{b}}\right.$, $\left.\mathrm{S}-\mathrm{CH}_{2}, \mathrm{CH}_{2}-\mathrm{O}\right), 2.41\left(\mathrm{~s}, 6 \mathrm{H}, 2 \mathrm{CH}_{3}\right), 1.20\left(\mathrm{t}, J=7.1 \mathrm{~Hz}, 3 \mathrm{H}, \mathrm{CH}_{3}\right) .{ }^{13} \mathrm{C} \mathrm{NMR}(75$ MHz, Acetone- $\left.d_{6}\right) ; \delta$ (ppm) 166.22, 166.22, 152.08, 152.08, 152.08, 145.49, $145.49,145.49,128.49,128.49,127.10,127.10,126.19,126.19,121.96,115.01$, 100.80, 59.06, 58.84, 27.28, 17.94, 13.67; HRMS (ESI): Calc for $\mathrm{C}_{22} \mathrm{H}_{22} \mathrm{~N}_{4} \mathrm{NaO}_{2} \mathrm{~S}$ $(\mathrm{M}+\mathrm{Na})^{+}:$429. 1407. Found: 429.1410.

Ethyl 2-(((1H-benzo[d]imidazol-2-yl) methyl)

thio)-6-methyl-4-(p-tolyl)-1,4-dihydropyrimidine-5-carboxylate $6 n$

Yield $=55 \%,{ }^{1} \mathrm{H}$ NMR $\left(300 \mathrm{MHz}, \mathrm{CDCl}_{3}\right) \delta(\mathrm{ppm}) 7.75-6.97\left(\mathrm{~m}, 8 \mathrm{H}, \mathrm{H}_{\mathrm{Ar}}\right)$, $5.76(\mathrm{~s}, 1 \mathrm{H}, \mathrm{CH}), 4.55\left(\mathrm{~d}, \mathrm{~J}_{a b}=14.7 \mathrm{~Hz}, 1 \mathrm{H}_{\mathrm{a}}, \mathrm{S}-\mathrm{CH}_{2}\right), 4.28-4.03\left(\mathrm{~m}, 3 \mathrm{H}, \mathrm{H}_{\mathrm{b}}\right.$, 
$\mathrm{CH}_{2}-\mathrm{O}$ ), $2.41\left(\mathrm{~s}, 6 \mathrm{H}, 2 \mathrm{CH}_{3}\right), 1.22\left(\mathrm{t}, J=6.8 \mathrm{~Hz}, 3 \mathrm{H}, \mathrm{CH}_{3}\right) ;{ }^{13} \mathrm{C}$ NMR (75 MHz, Acetone- $\left.\boldsymbol{d}_{\sigma}\right) . \delta(\mathrm{ppm}) 165.99,151.97,142.64,136.92,129.25,126.96,121.86$, 114.85, 59.38, 58.40, 20.30, 13.68; HRMS(ESI): Calc for $\mathrm{C}_{23} \mathrm{H}_{24} \mathrm{~N}_{4} \mathrm{NaO}_{2} \mathrm{~S}(\mathrm{M}+\mathrm{Na})^{+}$: 443.2035. Found: 443.2038.

\subsubsection{Biological Methods}

In this study, the evaluation of antibacterial activity was carried out according to the macro dilution technique reported by Marc et al. [27] and Moroh et al. [28], with some modifications. A series of dilution in Muller Hinton broth of the synthetic chemicals was carried out followed by a colony count on agar medium. Thus a $1000 \mu \mathrm{g} / \mathrm{mL}$ mother solution of the chemical was prepared by dissolving $0.1 \mathrm{~g}$ of powder of each synthetic chemical in $100 \mathrm{ml}$ of a DMSO/water mixture (50/50). The mother solution of $1000 \mu \mathrm{g} / \mathrm{mL}$ of the DMSO/water mixture (50/50) was incorporated into Muller Hinton broth in six test tubes to make final dilutions with concentrations ranging from: $500 ; 250 ; 125 ; 62.5 ; 31.250$ to 15.6250 $\mu \mathrm{g} / \mathrm{mL}$ with a dilution factor of $1 / 2$. Each tube contained $10 \mathrm{~mL}$ of the mother chemical-Muller Hinton broth mixture. Control tubes, i.e. without synthetic chemicals and containing only Muller Hinton broth were also prepared. All these solutions (media without synthetic chemicals and media with synthetic chemicals) were sterilized in an autoclave for $15 \mathrm{~min}$ at $121^{\circ} \mathrm{C}$. Then a bacterial inoculum estimated at $106 \mathrm{CFU} / \mathrm{mL}$ was prepared from a 16 hour-old colony of multidrug resistant strains of $E$. coli and $S$. aureus and adjusted by opacimeter with the standard Mac Farland. All the media (tests and controls) were inoculated with $0.2 \mathrm{ml}$ of the inoculums, then incubated at $37^{\circ} \mathrm{C}$ for 18 to 24 hours. The tests were repeated 3 times. Then the contents of the different tubes were transferred into Petri dishes containing $15 \mathrm{~mL}$ of Mueller-Hinton agar. This operation consisted of taking $1 \mathrm{~mL}$ of the broth contained in the tubes and added to $9 \mathrm{~mL}$ of sterile distilled water in order to have a $10^{-1}$ dilution. $1 \mathrm{~mL}$ of the $10^{-1}$ dilution is taken again and added to $9 \mathrm{~mL}$ of sterile distilled water to obtain the $10^{-2}$ dilution. This operation was repeated until to get a $10^{-4}$ dilution solution. A bactericidal test was carried out by inoculating $0.1 \mathrm{~mL}$ of dilutions of $10^{\circ}, 10^{-1}$, $10^{-2}, 10^{-3}$ and $10^{-4}$ by spreading on $15 \mathrm{~mL}$ of Mueller-Hinton agar, in a Petri box and incubated at $37^{\circ} \mathrm{C}$.

This operation was repeated three (3) times for each compound and each germ. After 24 hours of observation, the count of the colony count was done by direct counting. It is expressed as a percentage inhibition relative to the control. The method of calculation of the percentage inhibition could be summarized by the following formula:

$$
\begin{aligned}
& \text { Rate of inhibition }(\mathrm{Ti})(\%) \\
& =\left(1-\frac{\text { mean number of colonies for a given concentration }(\mathrm{CX})}{\text { mean number of colonies for a growth indicator }(\mathrm{TC})}\right) \times 100 \\
& \text { Rate of inhibition }(\mathrm{Ti})(\%)=\left(1-\frac{\mathrm{N}(\mathrm{CX})}{\mathrm{N}(\mathrm{TC})}\right) \times 100
\end{aligned}
$$


MIC: This is the lowest concentration of synthetic chemical that inhibits $99 \%$ of bacteria calculated compared to the control.

The $\mathrm{IC}_{50}$ was determined graphically from the sensitivity curve as a function of the concentrations of the synthetic chemicals.

\section{Results and Discussion}

\subsection{Chemistry}

The synthesis of the new thiopyrimidine molecules (6a-n) was achieved by reacting 2-thiopyrimidines (4) with benzyl halides or 2-(chloromethyl)-1H-benzimidazole in the presence of a base [29] (Scheme 1). The starting 2-thiopyridines were obtained by a stereospecific process between ethyl acetoacetate, thiourea and $p$-alkylbenzaldehydes under reflux of ethanol in the presence of hydrochloric acid [16]. From the two precursors, we got twelve new 2-benzylthio-pyrimidines (6a-1) by nucleophilic substitution reaction between 2-thiopyrimidines (4) and benzyl halides in dimethylformamide (DMF) in the presence of potassium carbonate $\left(\mathrm{K}_{2} \mathrm{CO}_{3}\right)$ as base. The originality of these compounds lied in introduction of diverse groups on the aromatic ring such as methyl $(\mathrm{Me})$, fluorine $(\mathrm{F})$, chlorine $(\mathrm{Cl})$, methyl carboxylate $\left(\mathrm{CO}_{2} \mathrm{Me}\right)$ and nitro $\left(\mathrm{NO}_{2}\right)$. The molecules were obtained with yields between $50 \%$ - 95\%. Likewise, for the benzimidazole derivatives $(6 \mathrm{~m}, 6 \mathrm{n})$, we obtained them by interaction of 2-thiopyrimidines $(4 \mathrm{a}, 4 \mathrm{~b})$ with 2-(chloromethyl)-1H-benzimidazole in tetrahydrofuran (THF) in the presence of triethylamine such as base. Both compounds were obtained with $60 \%$ and $55 \%$ yields respectively. The ${ }^{1} \mathrm{H}$ NMR spectrum of the 2-benzylthio-pyrimidines (6a-1) revealed the presence of two doublets between 4.13 and 5.43 ppm that we attributed to the two protons of the methylene group of benzyl bond to the sulfur atom $\left(\mathrm{S}-\mathrm{CH}_{2}\right)$. The appearance of twice protons in two doublets could be explained by the tetrahedral geometry of the molecule. Protons behave as if they were neighbors to an asymmetric carbon. For compounds $6 \mathrm{~m}$ and $6 \mathrm{n}$, the ${ }^{1} \mathrm{H}$ NMR spectrum revealed the presence of a doublet at $4.53 \mathrm{ppm}$ corresponding to a proton of the methylene group $\left(\mathrm{CH}_{2}-\mathrm{S}\right)$ and the second proton appeared between 4.28 and $4.03 \mathrm{ppm}$ as a multiplet.

\subsection{Biology}

The average of the results obtained is given in Table 1.

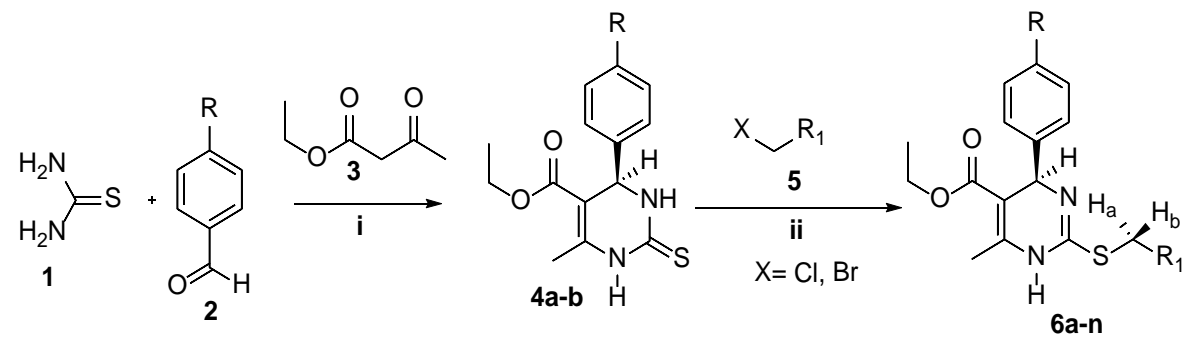

Scheme 1. Synthesis of compounds 6a-n. Reagents and operating conditions: (i): HCl/ethanol (reflux); (ii) $\mathrm{K}_{2} \mathrm{CO}_{3} / \mathrm{DMF}$ or $\mathrm{Et}_{3} \mathrm{~N} / \mathrm{THF}$. 
Table 1. Antibacterial activity of 2-benzylthiopyrimidines and 2-benzimidazolylmethylthiopyrimidines derivatives (rate of inhibition $(\mathrm{Ti})$ and $\mathrm{IC}_{50}$ ).

\begin{tabular}{|c|c|c|c|c|c|}
\hline \multirow{2}{*}{\multicolumn{2}{|c|}{$\begin{array}{l}\text { Concentration of compounds } \\
\qquad(\mu \mathrm{g} / \mathrm{mL})\end{array}$}} & \multicolumn{2}{|c|}{ S. aureus 1174} & \multicolumn{2}{|c|}{ E. coli 1178} \\
\hline & & $\operatorname{Ti}(\%)$ & $\begin{array}{c}\mathrm{IC}_{50} \\
(\mu \mathrm{g} / \mathrm{mL})\end{array}$ & $\operatorname{Ti}(\%)$ & $\begin{array}{c}\mathrm{IC}_{50} \\
(\mu \mathrm{g} / \mathrm{mL})\end{array}$ \\
\hline \multirow{6}{*}{$6 a$} & 500 & 91.16 & \multirow{6}{*}{26.04} & 82.35 & \multirow{6}{*}{29.68} \\
\hline & 250 & 83.33 & & 76.47 & \\
\hline & 125 & 75 & & 70.58 & \\
\hline & 62.5 & 66.66 & & 58.82 & \\
\hline & 31.25 & 58.33 & & 52.94 & \\
\hline & 15.625 & 33.33 & & 23.52 & \\
\hline \multirow{6}{*}{$6 \mathrm{~b}$} & 500 & 83.33 & \multirow{6}{*}{61.07} & 82.35 & \multirow{6}{*}{57.06} \\
\hline & 250 & 75 & & 70.58 & \\
\hline & 125 & 66.66 & & 64.7 & \\
\hline & 62.5 & 50 & & 52.94 & \\
\hline & 31.25 & 41.66 & & 35.29 & \\
\hline & 15.625 & 25 & & 23.52 & \\
\hline \multirow{6}{*}{$6 c$} & 500 & 100 & \multirow{6}{*}{25.83} & 70.58 & \multirow{6}{*}{116.55} \\
\hline & 250 & 100 & & 58.82 & \\
\hline & 125 & 100 & & 52.94 & \\
\hline & 62.5 & 91.66 & & 35.29 & \\
\hline & 31.25 & 51.66 & & 35.29 & \\
\hline & 15.625 & 41.66 & & 29.41 & \\
\hline \multirow{6}{*}{$6 d$} & 500 & 91.66 & \multirow{6}{*}{15.625} & 76.47 & \multirow{6}{*}{45.86} \\
\hline & 250 & 91.66 & & 76.47 & \\
\hline & 125 & 83.33 & & 70.58 & \\
\hline & 62.5 & 83.33 & & 52.94 & \\
\hline & 31.25 & 66.66 & & 47.05 & \\
\hline & 15.625 & 50 & & 41.17 & \\
\hline \multirow{6}{*}{$6 \mathrm{~h}$} & 500 & 91.66 & \multirow{6}{*}{15.625} & 94.11 & \multirow{6}{*}{13.02} \\
\hline & 250 & 91.66 & & 88.23 & \\
\hline & 125 & 91.66 & & 82.35 & \\
\hline & 62.5 & 66.66 & & 76.47 & \\
\hline & 31.25 & 66.66 & & 70.58 & \\
\hline & 15.625 & 50 & & 52.94 & \\
\hline \multirow{6}{*}{$6 j$} & 500 & 83 & \multirow{6}{*}{41.3} & 76.47 & \multirow{6}{*}{55.15} \\
\hline & 250 & 75 & & 70.58 & \\
\hline & 125 & 66.66 & & 64.7 & \\
\hline & 62.5 & 58.33 & & 52.94 & \\
\hline & 31.25 & 48.5 & & 47.05 & \\
\hline & 15.625 & 41.66 & & 35.29 & \\
\hline
\end{tabular}




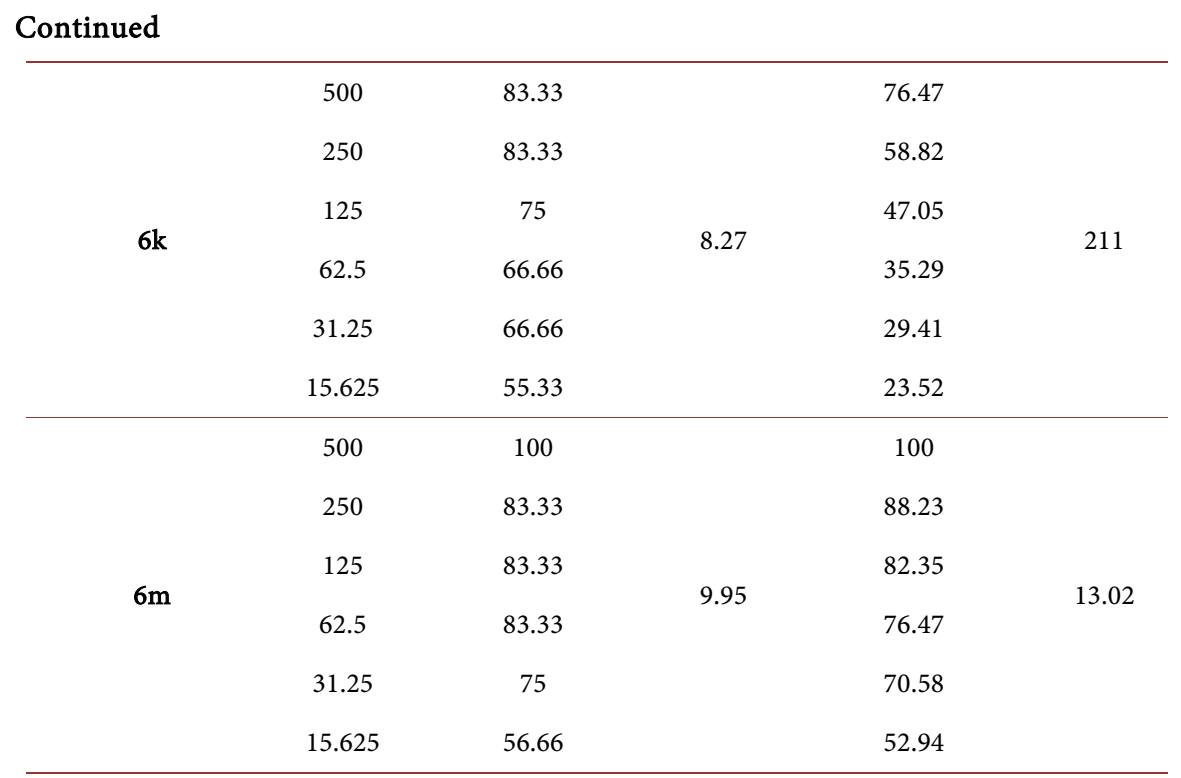

Data processing allowed to plot the sensitivity curves of the germs (inhibition rate as a function of the product concentrations) and the concentration at which the substances inhibit the germs by half $\left(\mathrm{IC}_{50}\right)$ was determined graphically.

Multidrug-resistant strains of both $E$. coli and $S$. aureus showed good sensitivity to the eight 2-thiopyrimidine derivatives with a better sensitivity to $S$. aureus. Compounds $6 \mathrm{c}, 6 \mathrm{~d}, 6 \mathrm{~h}$ and $6 \mathrm{~m}$ were more active against $S$. aureus, while compounds $6 \mathrm{~h}$ and $6 \mathrm{~m}$ were more active against $E$. coli. The presence of the benzyl and benzimidazolyl groups in position-2 of 2-thiopyrimidines demonstrated good antibacterial activity. In this series of compounds, the presence of the nitro group $\left(\mathrm{NO}_{2}\right)$ at the 3-position and methyl group at the 4-position of the benzyl enhanced the biological activity on $S$. aureus. Likewise, the attachment of chloride group at the 4-position of the benzyl enhanced the inhibition activity on $E$. coli. Introduction of benzimidazole ring to the sulfur atom at position-2 of the pyrimidine ring enhanced the biological activity against both $S$. aureus and $E$. coli. Compound $6 \mathrm{c}$ with the nitro group in position-3 on the phenyl was more active on $S$. aureus with a MIC $=125 \mu \mathrm{g} / \mathrm{mL}$ while compound $6 \mathrm{~m}$ was more active on both $S$. aureus and $E$. coli with a MIC $=500 \mu \mathrm{g} / \mathrm{mL}$.

\section{Conclusion}

In this work, it is showed through a number of experimental evidence that between fourteen (14) new 2-thiopyrimidines derivatives (6a-n) obtained in yields ranging from $50 \%$ to $94 \%$, eight of them were submitted to antibacterial tests on multidrug-resistant strains of $E$. coli and $S$. aureus. They showed good activities on both resistant bacteria. Moreover, compounds $6 \mathrm{c}(\mathrm{MIC}=125 \mu \mathrm{g} / \mathrm{mL})$ and $6 \mathrm{~m}(\mathrm{MIC}=500 \mu \mathrm{g} / \mathrm{mL})$ were efficiently active on $S$. aureus and similarly compound $6 \mathrm{~m}(\mathrm{MIC}=500 \mu \mathrm{g} / \mathrm{mL})$ was active on $E$. coli. Furthermore, these results validated our approach on the design of new 2-thiopyrimidine derivatives by the attachment of benzyl and benzimidazolyl groups in the 2 position. This ap- 
proach could be an addressing contribution to the development of new antibiotics. The next challenge to access antibiotic-resistant is to bring the active compounds into a large spectrum of bacteria.

\section{Acknowledgements}

We wish to thank the laboratory (Laboratoire de Méthodologie et Synthèse de Produits Naturels) of the University of Quebec in Montreal (Canada) and the Laboratory LG2A of Jules Verne Picardie University (France) for providing us the chemical reagents and material for the spectroscopic analyzes.

\section{Conflicts of Interest}

The authors declare no conflicts of interest regarding the publication of this paper.

\section{References}

[1] Eicher, T., Hauptmann, S. and Speicher, A. (2003) The Chemistry of Heterocycles: Structures, Reactions, Synthesis, and Applications. John Wiley \& Sons, Hoboken. https://doi.org/10.1002/352760183X

[2] Chiang, A.N., Valderramos, J.-C., Balachandran, R., Chovatiya, R.J., Mead, B.P., Schneider, C., Bell, S.L., Klein, M.G., Huryn, D.M., Chen, X.S., et al. (2009) Select Pyrimidinones Inhibit the Propagation of the Malarial Parasite, Plasmodium falciparum. Bioorganic \& Medicinal Chemistry, 17, 1527-1533. https://doi.org/10.1016/j.bmc.2009.01.024

[3] Clercq, E.D., Sakuma, T., Baba, M., Pauwels, R., Balzarini, J., Rosenberg, I. and Holý, A. (1987) Antiviral Activity of Phosphonylmethoxyalkyl Derivatives of Purine and Pyrimidines. Antiviral Research, 8, 261-272. https://doi.org/10.1016/S0166-3542(87)80004-9

[4] Rajanarendar, E., Reddy, M.N., Murthy, K.R., Reddy, K.G., Raju, S., Srinivas, M., Praveen, B. and Rao, M.S. (2010) Synthesis, Antimicrobial, and Mosquito Larvicidal Activity of 1-aryl-4-methyl-3,6-bis-(5-methylisoxazol-3-yl)-2-thioxo-2,3,6,10b-tetrahydro- $1 H$-pyrimido[5,4-c] quinolin-5-ones. Bioorganic \& Medicinal Chemistry Letters, 20, 6052-6055. https://doi.org/10.1016/j.bmcl.2010.08.060

[5] Virsodia, V., Pissurlenkar, R.R.S., Manvar, D., Dholakia, C., Adlakha, P., Shah, A. and Coutinho, E.C. (2008) Synthesis, Screening for Antitubercular Activity and 3D-QSAR Studies of Substituted N-phenyl-6-methyl-2-oxo-4-phenyl-1,2,3,4-tetrahydro-pyrimidine-5-carboxamides. European Journal of Medicinal Chemistry, 43, 2103-2115. https://doi.org/10.1016/j.ejmech.2007.08.004

[6] Regnier, G., Canevari, R., Le Douarec, J., Holstorp, S. and Daussy, J. (1972) Triphenylpropylpiperazine Derivatives as New Potent Analgetic Substances. Journal of Medicinal Chemistry, 15, 295-301. https://doi.org/10.1021/jm00273a600

[7] Farghaly, A.M., AboulWafa, O.M., Elshaier, Y.A., Badawi, W.A., Haridy, H.H. and Mubarak, H.A. (2019) Design, Synthesis, and Antihypertensive Activity of New Pyrimidine Derivatives Endowing New Pharmacophores. Medicinal Chemistry Research, 28, 360-379. https://doi.org/10.1007/s00044-019-02289-6

[8] Wang, Z., Gao, Y., He, L., Sun, S., Xia, T., Hu, L., Yao, L., Wang, L., Li, D., Shi, H. and Liao, X. (2021) Structure-Based Design of Highly Potent Toll-Like Receptor 7/8 Dual Agonists for Cancer Immunotherapy. Journal of Medicinal Chemistry, 64 
7507-7532. https://doi.org/10.1021/acs.jmedchem.1c00179

[9] Maquoi, E., Sounni, N. E., Devy, L., Olivier, F., Frankenne, F., Krell, H.-W., Grams, F., Foidart, J.-M. and Noël, A. (2004) Anti-Invasive, Antitumoral, and Antiangiogenic Efficacy of a Pyrimidine-2,4,6-trione Derivative, an Orally Active and Selective Matrix Metalloproteinases Inhibitor. Clinical Cancer Research, 10, 4038-4047. https://doi.org/10.1158/1078-0432.CCR-04-0125

[10] Huang, M., Wang, Y., Collins, M., Mitchell, B.S. and Graves, L.M. (2002) A77 1726 Induces Differentiation of Human Myeloid Leukemia K562 Cells by Depletion of Intracellular CTP Pools. Molecular Pharmacology, 62, 463-472. https://doi.org/10.1124/mol.62.3.463

[11] Von Bubnoff, N., Veach, D.R., Miller, W.T., Li, W., Sänger, J., Peschel, C., Bornmann, W.G., Clarkson, B. and Duyster, J. (2003) Inhibition of Wild-Type and Mutant Bcr-Abl by Pyrido-Pyrimidine-Type Small Molecule Kinase Inhibitors. Cancer Research, 63, 6395-6404.

[12] Mojtahedi, M.M., Saidi, M.R., Shirzi, J.S. and Bolourtchian, M. (2002) Microwave Promoted Efficient Synthesis of Substituted Uracils and Thiouracils under Solvent-Free Conditions. Synthetic Communications, 32, 851-855. https://doi.org/10.1081/SCC-120002693

[13] Sondhi, S.M., Goyal, R.N., Lahoti, A.M., Singh, N., Shukla, R. and Raghubir, R. (2005) Synthesis and Biological Evaluation of 2-Thiopyrimidine Derivatives. Bioorganic \& Medicinal Chemistry, 13, 3185-3195. https://doi.org/10.1016/j.bmc.2005.02.047

[14] Sochacka, E. and Frątczak, I. (2004) Efficient Desulfurization of 2-Thiopyrimidine Nucleosides to the Corresponding 4-Pyrimidinone Analogues Using Trans-2-(phenylsulfonyl)-3-phenyloxaziridine. Tetrahedron Letters, 45, 6729-6731. https://doi.org/10.1016/j.tetlet.2004.07.052

[15] Salina, E., Ryabova, O., Kaprelyants, A. and Makarov, V. (2014) New 2-Thiopyridines as Potential Candidates for Killing both Actively Growing and Dormant Mycobacterium tuberculosis Cells. Antimicrobial Agents and Chemotherapy, 58, 55-60. https://doi.org/10.1128/AAC.01308-13

[16] Biginelli, P. and Gazz, P. (1893) Synthesis of 3,4-Dihydropyrimidin-2(1H)-Ones. Gazzetta Chimica Italiana, 23, 360-416.

[17] Bamba, F., Jin, J., Chaudhary, A.S., Tai, P.C. and Wang, B. (2021) Design, Synthesis, and Biological Evaluation of Pyrimidine Analogs as SecA Inhibitors. Medicinal Chemistry Research, 30, 1334-1340. https://doi.org/10.1007/s00044-021-02717-6

[18] Bamba, F., Jin, J., Tai, P.C. and Wang, B. (2020) Synthesis and Biological Evaluation of Novel 4-oxo-5-cyano Thiouracil Derivatives as SecA Inhibitors. Heterocyclic Communications, 26, 76-83. https://doi.org/10.1515/hc-2020-0100

[19] Maddila, S., Gorle, S., Seshadri, N., Lavanya, P. and Jonnalagadda, S.B. (2016) Synthesis, Antibacterial and Antifungal Activity of Novel Benzothiazole Pyrimidine Derivatives. Arabian Journal of Chemistry, 9, 681-687. https://doi.org/10.1016/j.arabjc.2013.04.003

[20] Shigeta, S., Mori, S., Watanabe, F., Takahashi, K., Nagata, T., Koike, N., Wakayama, T. and Saneyoshi, M. (2002) Synthèse et activités anti-herpèsvirus d'analogues nucléosidiques 5-Alkyl-2-Thiopyrimidine. Antiviral Chemistry and Chemotherapy, 13, 67-82. https://doi.org/10.1177\%2F095632020201300201

[21] Mokale, S. N., Shinde, S. S., Elgire, R. D., Sangshetti, J. N. and Shinde, D. B. (2010) Synthesis and Anti-Inflammatory Activity of Some 3-(4,6-disubtituted-2-thioxo-1,2,3,4tetrahydropyrimidin-5-yl) Propanoic Acid Derivatives. Bioorganic \& Medicinal Chemistry Letters, 20, 4424-4426. https://doi.org/10.1016/j.bmcl.2010.06.058 
[22] Pathak, A.K., Pathak, V., Seitz, L.E., Suling, W.J. and Reynolds, R.C. (2004) Antimycobacterial Agents. 1. Thio Analogues of Purine. Journal of Medicinal Chemistry, 47, 273 276. https://doi.org/10.1021/jm030389b

[23] Lal, K., Paliwal, L. and Bagade, M. (2018) Synthesis, Molecular Docking and Pharmacological Study of Some New Thiopyrimidine Derivatives. Materials Today: Proceedings, 5, 15354-15360. https://doi.org/10.1016/j.matpr.2018.05.017

[24] Tonelli, M., Paglietti, G., Boido, V., Sparatore, F., Marongiu, F., Marongiu, E., La Colla, P. and Loddo, R. (2008) Antiviral Activity of Benzimidazole Derivatives. I. Antiviral Activity of 1-Substituted-2-[(Benzotriazol-1/2-yl)methyl]benzimidazoles. Chemistry \& Biodiversity, 5, 2386-2401. https://doi.org/10.1002/cbdv.200890203

[25] Ouattara, M., Sissouma, D., Yavo, W. and Kone, M.W. (2015) Synthèse et criblage antiplasmodial de quelques benzimidazolyl-chalcones. International Journal of Biological and Chemical Sciences, 9, 1697-1710.

https://doi.org/10.4314/ijbcs.v9i3.48

[26] Ouattara, M., Sissouma, D., Koné, M., Menan, H., Touré, S. and Ouattara, L. (2011) Synthesis and Anthelmintic Activity of Some Hybrid Benzimidazolyl-Chalcone Derivatives. Tropical Journal of Pharmaceutical Research, 10, 767-775. https://doi.org/10.4314/tjpr.v10i6.10

[27] Marc, T., Gérard, W. and Denis, L. (2001) Classification des anti-inflamatoires dans Guide pharmacologie. In: Guide Pharmacologie. Etudiants et professionnels paramédicaux, 4th Edition, 126-128.

[28] Moroh, J.-L.A., Bahi, C., Dje, K., Loukou, Y.G. and Guede-Guina, F. (2008). Study of the Antibacterial Activity of Morinda Morindoides (Baker) Milne-Redheat (Rubiaceae) Acetatique Extract (ACE) on In Vitro Growth of Escherichia Coli Strains. Bullentin de la société Royale des Sciences de Liège, 77.

[29] Severina, H.I., Skupa, O.O., Voloshchuk, N.I., Suleiman, M.M. and Georgiyants, V.A. (2019) Synthesis and Anticonvulsant Activity of 6-methyl-2-((2-oxo-2-arylethyl)thio)pyrimidin-4(3H)-one Derivatives and Products of Their Cyclization. Pharmacia, 66, 141-146. https://doi.org/10.3897/pharmacia.66.e38137 